VERSITA

\author{
TATRA \\ MOUNTaiNs \\ Mathematical Publications \\ DOI: $10.2478 / \mathrm{v} 10127-011-0033-1$ \\ Tatra Mt. Math. Publ. 50 (2011), 13-24
}

\title{
A PREDATOR-PREY MODEL WITH ALLEE EFFECT AND FAST STRATEGY EVOLUTION DYNAMICS OF PREDATORS USING HAWK AND DOVE TACTICS
}

\author{
Jitka Kühnová - LenkA PřIBYlová
}

\begin{abstract}
In this work we present the predator-prey model with Allee effect and Hawk and Dove tactics in fighting over caught prey implemented as fast strategy evolution dynamics. We extend the work of Auger, Parra, Morand and Sánchez (2002) using the prey population embodying Allee effect and analogously to this work we get two connected submodels with polymorphic and monomorphic predator population. We get much richer dynamics, in each submodel we find local bifurcations (saddle-node, supercritical Hopf caused by Allee effect and Bogdanov-Takens) and a global bifurcation of limit cycles caused by the strategy evolution that is not possible in any of the submodels that can lead to a bluesky extinction of both populations.
\end{abstract}

\section{The model}

In our model, we expect the prey is limited by a carrying capacity and a threshold of survivance, which means that prey becomes extinct when its density is lower than $\theta$. It is typical for a lot of populations - only few could grow up from just one or two individuals. This so called Allee effect is not considered in [1.

The predator population is divided into two types related to the predator behaviour. Whenever two predators meet after catching the prey, they choose their behaviour strategy. They can initiate aggressive behaviour and fight over the prey (we expect they are equally likely to be injured) or they retreat. The first predators are so called Hawks, the second are Doves. From the game theory it is known as the Hawk and Dove game. Whenever two Hawk predators meet, they both initiate aggressive behaviour, the conflict results and one of them gets the prey (gain $G>0)$. The cost of the conflict $(C$ - given effort and got injuries)

(C) 2011 Mathematical Institute, Slovak Academy of Sciences.

2010 Mathematics Subject Classification: 34C60, 34C23, 92D50.

Keywords: prey-predator model, Allee effect, strategy evolution, bifurcation, Hawk-Dove evolution.

The research was supported by the internal grant of Masaryk University MUNI/A/1060/2009. 


\section{JITKA KÜHNOVÁ — LENKA PŘIBYLOVÁ}

reduces individual fitness of the other by some positive constant value $C$. When a Hawk meets a Dove, the Dove immediately retreats and the Hawk obtains the resource and when two Doves meet the resource is shared equally between them. The fitness payoffs for the Hawk-Dove game can be summarized according to the following matrix

$$
\boldsymbol{A}=\left(\begin{array}{cc}
\frac{G-C}{2} & G \\
0 & \frac{G}{2}
\end{array}\right)
$$

Let the function $p$ in time $t$ be the function of the number of predators which is the sum of Hawk $p_{H}(t)$ and Dove $p_{D}(t)$ predators. Let $x(t)$ and $y(t)$ be the proportions of predators with Hawks and Doves tactics

$$
x(t)=\frac{p_{H}(t)}{p(t)}, \quad y(t)=\frac{p_{D}(t)}{p(t)}=1-x(t),
$$

respectively.

Now we need to create a model in fast time scale by using replicators equations

$$
\begin{aligned}
& \frac{\mathrm{d} x}{\mathrm{~d} \tau}=x\left(\Delta_{H}-\Delta\right), \\
& \frac{\mathrm{d} y}{\mathrm{~d} \tau}=y\left(\Delta_{D}-\Delta\right),
\end{aligned}
$$

where $\Delta_{H}$ is the gain of an individual always using the Hawk strategy, $\Delta_{D}$ is the gain of an individual always using the Dove strategy and $\Delta$ is the average gain of an individual playing the two tactics. Using the fact that $x+y=1$ in any time $t$ and after some algebra we get single equation:

$$
\frac{\mathrm{d} x}{\mathrm{~d} \tau}=\frac{x}{2}(1-x)(G-C x) .
$$

The stationary points are 0,1 a $G / C=x^{*}$. When $G<C$ we denote $x^{*}=\frac{G}{C}$. This stationary point is asymptotically stable, population of the predators is polymorphic with proportion $G / C$ Hawks and $1-G / C$ Doves. When $G>C$ we denote $x^{*}=1$, and this stationary point is asymptotically stable, population of the predators is monomorphic (there are only Hawks).

For model in slow time scale we need the equation for population of the prey. Because of Allee effect in prey population, intraspecific competition and constant harvesting by predator we get

$$
\frac{\mathrm{d} n}{\mathrm{~d} t}=r n\left(\frac{n}{\theta}-1\right)\left(1-\frac{n}{K}\right)-a n p,
$$

where $\theta$ is the threshold of survivance, $r$ is the growth rate of prey population, $K$ is the carrying capacity and $a$ is a predation force parameter. 


\section{A PREDATOR-PREY MODEL WITH ALLEE EFFECT}

For equations of predator populations we use assumptions from [1] and we get

$$
\begin{aligned}
\frac{\mathrm{d} p_{H}}{\mathrm{~d} t} & =-b p_{H}+\left(\alpha\left(\frac{G-C}{2}\right) \frac{p_{H}}{p}+\alpha G \frac{p_{D}}{p}\right) p_{H}, \\
\frac{\mathrm{d} p_{D}}{\mathrm{~d} t} & =-b p_{D}+\alpha \frac{G}{2} \frac{p_{D}}{p} p_{D},
\end{aligned}
$$

where $b$ is the mortality rate of the predator (without prey predator becomes extinct), $\alpha$ is a conversion coefficient of gain and cost into biomass of predators. All coefficients are positive.

We assume that the model in fast time scale is established in the stationary point $x^{*}$ and by using the theory of aggregated model [1] we get:

$$
\begin{aligned}
& \frac{\mathrm{d} n}{\mathrm{~d} t}=r n\left(\frac{n}{\theta}-1\right)\left(1-\frac{n}{K}\right)-a n p, \\
& \frac{\mathrm{d} p}{\mathrm{~d} t}=-b p+\frac{\alpha G}{2} p-\frac{\alpha C}{2}\left(x^{*}\right)^{2} p .
\end{aligned}
$$

If the gain depends on the prey density as $G(n)=a n$, we obtain two different aggregated models:

Model I, $n<\frac{C}{a}$,

$$
\begin{aligned}
& \frac{\mathrm{d} n}{\mathrm{~d} t}=r n\left(\frac{n}{\theta}-1\right)\left(1-\frac{n}{K}\right)-a n p, \\
& \frac{\mathrm{d} p}{\mathrm{~d} t}=-b p+\frac{\alpha a}{2} n p-\frac{\alpha a^{2}}{2 C} n^{2} p .
\end{aligned}
$$

Model II, $n>\frac{C}{a}$,

$$
\begin{aligned}
& \frac{\mathrm{d} n}{\mathrm{~d} t}=r n\left(\frac{n}{\theta}-1\right)\left(1-\frac{n}{K}\right)-a n p \\
& \frac{\mathrm{d} p}{\mathrm{~d} t}=-b p+\frac{\alpha a}{2} n p-\frac{\alpha C}{2} p .
\end{aligned}
$$

Now denote:

$$
\begin{aligned}
P(n) & =r\left(\frac{n}{\theta}-1\right)\left(1-\frac{n}{K}\right), & & \\
Q(n) & =-b+\frac{\alpha a}{2} n-\frac{\alpha a^{2}}{2 C} n^{2} & & \text { for } n<\frac{C}{a}, \\
& =-b+\frac{\alpha a}{2} n-\frac{\alpha C}{2} & & \text { for } n>\frac{C}{a} .
\end{aligned}
$$

We get:

$$
\begin{aligned}
& \frac{\mathrm{d} n}{\mathrm{~d} t}=n[P(n)-a n], \\
& \frac{\mathrm{d} p}{\mathrm{~d} t}=Q(n) p .
\end{aligned}
$$




\section{JITKA KÜHNOVÁ — LENKA PŘIBYLOVÁ}

\section{Analysis of the model}

Nullclines of the system:

- nullclines for both models: $n=0, p=0, p=\frac{1}{a} P(x)$.

- nullclines for the first model: $n_{1,2}^{*}=\frac{C}{2 a}\left(1 \mp \sqrt{1-\frac{8 b}{\alpha C}}\right)$.

- nullcline for the second model: $n_{3}^{*}=\frac{C}{a}+\frac{2 b}{\alpha a}$.

For $n_{1}^{*}, n_{2}^{*}, n_{3}^{*}$ it is always true:

$$
0<n_{1}^{*} \leq \frac{C}{2 a} \leq n_{2}^{*}<\frac{C}{a}<n_{3}^{*} .
$$

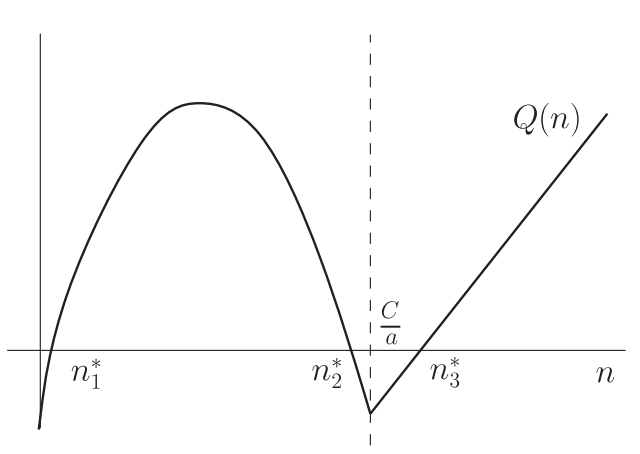

FiguRe 1

Function $Q(n)$.

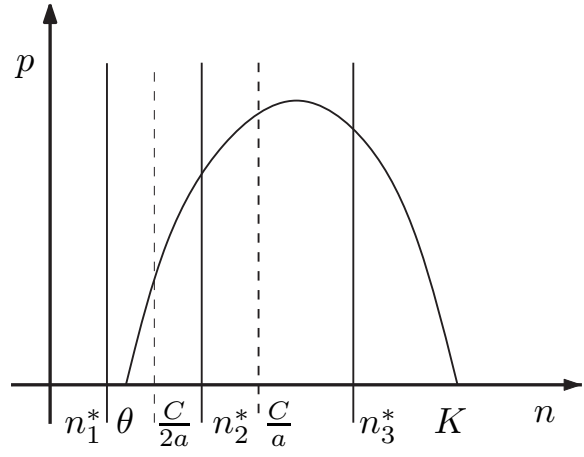

Figure 2

Phase portrait of the combined model.

It is clear that, there are six stationary points:

$$
(0,0),(\theta, 0),(K, 0),\left(n_{1}^{*}, p_{1}^{*}\right),\left(n_{2}^{*}, p_{2}^{*}\right) \text { and }\left(n_{3}^{*}, p_{3}^{*}\right) .
$$

The last three points exist only when

$$
n_{1}^{*}, n_{2}^{*}, n_{3}^{*} \in(\theta, K) .
$$

For finding out the type and stability of all stationary points it is necessary to examine Jacobian matrix for all of them. The general Jacobian matrix

$$
\boldsymbol{J}\left(n^{*}, p^{*}\right)=\left(\begin{array}{cc}
P\left(n^{*}\right)-a p^{*}+n^{*} P^{\prime}\left(n^{*}\right) & -a n^{*} \\
p^{*} Q^{\prime}\left(n^{*}\right) & Q\left(n^{*}\right)
\end{array}\right)
$$

1.(0,0): always stable node or focus. Because of that, in every situation there are trajectories that end at beginning. That means both the prey and the predator become extinct. 


\section{A PREDATOR-PREY MODEL WITH ALLEE EFFECT}

2. $(\theta, 0)$ : saddle for $\theta \in\left(0, n_{1}^{*}\right) \cup\left(n_{2}^{*}, n_{3}^{*}\right)$, unstable node or focus, otherwise.

3. $(K, 0)$ : saddle for $K \in\left(n_{1}^{*}, n_{2}^{*}\right) \cup\left(n_{3}^{*}, \infty\right)$, stable node or focus, otherwise.

4. $\left(n_{1}^{*}, p_{1}^{*}\right)$ : for $n_{1}^{*}<\frac{K+\theta}{2}$ unstable, for $n_{1}^{*}>\frac{K+\theta}{2}$ stable node or focus.

5. $\left(n_{2}^{*}, p_{2}^{*}\right)$ : always saddle.

6. $\left(n_{3}^{*}, p_{3}^{*}\right)$ : for $n_{3}^{*}<\frac{K+\theta}{2}$ unstable, for $n_{3}^{*}>\frac{K+\theta}{2}$ stable node or focus.

At the first sight there could be five different situations:

1. only $(0,0)$ is locally stable stationary point,

2. $(0,0)$ and $(K, 0)$ are locally stable stationary points,

3. $(0,0)$ and $\left(n_{1}^{*}, p_{1}^{*}\right)$ are locally stable stationary points,

4. $(0,0),(K, 0)$ and $\left(n_{1}^{*}, p_{1}^{*}\right)$ are locally stable stationary points,

5 . $(0,0)$ and $\left(n_{3}^{*}, p_{3}^{*}\right)$ are locally stable stationary points.

The first two situations are not interesting - neither population survive, there is nothing to examine; the prey could not satisfy predator, predator becomes extinct and prey is stabilized on its carrying capacity. The third and the fifth situations have stable stationary point of coexistence of both populations, the fourth has either coexistence point or extinction of predator and prey stabilized on its carrying capacity.

From the analysis one could see that stationary points

$$
\left(n_{1}^{*}, p_{1}^{*}\right) \text { and }\left(n_{3}^{*}, p_{3}^{*}\right)
$$

could be stable or unstable focuses which implies there could be two Hopf bifurcations.

To examine stability of the limit cycle it is necessary to translate the system to the normal form of the Hopf bifurcation. First, we shift the system to the origin, second, we make linear transformation to the normal form and consequently we find the first Lyapunov coefficient whose sign determines the stability of the limit cycle.

Generally, our system (3) has the stationary point $\left(n^{*}, p^{*}\right)$. Let us transform the variables:

$$
\begin{aligned}
& n=\xi_{1}+n^{*}, \\
& p=\xi_{2}+p^{*} .
\end{aligned}
$$

We get the new system

$$
\boldsymbol{\xi}^{\prime}=\boldsymbol{f}(\boldsymbol{\xi})
$$

that could be rewritten as

$$
\boldsymbol{\xi}^{\prime}=\boldsymbol{A}_{0} \cdot \boldsymbol{\xi}+\boldsymbol{F}(\boldsymbol{\xi}), \quad \boldsymbol{\xi}=\left(\xi_{1}, \xi_{2}\right)^{T},
$$




\section{JITKA KÜHNOVÁ - LENKA PŘIBYLOVÁ}

where $\boldsymbol{A}_{0}$ is Jacobian matrix of the system (5) in the origin, $\operatorname{denote} \operatorname{det} \boldsymbol{A}_{0}=\omega^{2}$.

Now we change variables with matrix $\boldsymbol{T}$ of real eigenvectors of $\boldsymbol{A}_{0}$ :

$$
\begin{aligned}
\boldsymbol{\xi} & =\boldsymbol{T} \cdot \boldsymbol{\mu}, \\
\boldsymbol{\mu}^{\prime} & =\boldsymbol{T}^{-1} \boldsymbol{A}_{0} \boldsymbol{T} \boldsymbol{\mu}+\boldsymbol{T}^{-1} \boldsymbol{F}(\boldsymbol{T} \boldsymbol{\mu}), \\
\boldsymbol{P}(\boldsymbol{\mu}) & =\boldsymbol{T}^{-1} \boldsymbol{F}(\boldsymbol{T} \boldsymbol{\mu}), \\
\boldsymbol{\mu}^{\prime} & =\boldsymbol{J} \cdot \boldsymbol{\mu}+\boldsymbol{P}(\boldsymbol{\mu}) .
\end{aligned}
$$

$\boldsymbol{J}$ is in the real Jordan form, in detail

$$
\left(\begin{array}{l}
\mu_{1} \\
\mu_{2}
\end{array}\right)^{\prime}=\left(\begin{array}{cc}
0 & -\omega \\
\omega & 0
\end{array}\right)\left(\begin{array}{l}
\mu_{1} \\
\mu_{2}
\end{array}\right)+\left(\begin{array}{l}
P\left(\mu_{1}, \mu_{2}\right) \\
R\left(\mu_{1}, \mu_{2}\right)
\end{array}\right) .
$$

The stability of the limit cycle is determined by the first Lyapunov coefficient

$$
l_{1}(0)=\frac{1}{8 \omega} L_{1}+\frac{1}{8 \omega^{2}} L_{2}
$$

with

$$
\begin{aligned}
& L_{1}=P_{111}+P_{122}+R_{112}+R_{222}, \\
& L_{2}=P_{12}\left(P_{11}+P_{22}\right)-R_{12}\left(R_{11}+R_{22}\right)-P_{11} R_{11}+P_{22} R_{22},
\end{aligned}
$$

where the lower indices mean partial derivatives of $\boldsymbol{P}$ respective to components evaluated at $\boldsymbol{\mu}=\mathbf{0}$.

We have the eigenvectors of $\boldsymbol{A}_{0}$ :

and matrix $\boldsymbol{T}$

$$
\left(\begin{array}{c}
1 \\
-\frac{i \omega}{a n_{i}^{*}}
\end{array}\right), \quad\left(\begin{array}{c}
1 \\
\frac{i \omega}{a n_{i}^{*}}
\end{array}\right)
$$

$$
\left(\begin{array}{cc}
1 & 0 \\
0 & \frac{\omega}{a n_{i}^{*}}
\end{array}\right), \quad i=1,3
$$

For $\left(n_{1}^{*}, p_{1}^{*}\right)$ we have the functions $P\left(\mu_{1}, \mu_{2}\right), R\left(\mu_{1}, \mu_{2}\right)$ :

$$
\begin{aligned}
& P\left(\mu_{1}, \mu_{2}\right)=-\frac{r \mu_{1}{ }^{3}}{\theta K}+\frac{r\left(K+\theta-3 n_{1}^{*}\right)}{\theta K} \mu_{1}^{2}-\frac{\omega}{n_{1}^{*}} \mu_{1} \mu_{2}+\frac{r}{K \theta}\left(K+\theta-2 n_{1}^{*}\right) n_{1}^{*} \mu_{1}, \\
& R\left(\mu_{1}, \mu_{2}\right)=\frac{\alpha a}{2 C}\left[\left(C-2 a n_{1}^{*}\right) \mu_{1} \mu_{2}-a\left(\frac{a n_{1}^{*} p_{1}^{*}}{\omega}+\mu_{2}\right) \mu_{1}^{2}\right] .
\end{aligned}
$$




\section{A PREDATOR-PREY MODEL WITH ALLEE EFFECT}

After some algebra we get the first Lyapunov coefficient

$$
l_{1}(0)=-\frac{r \alpha a^{2} n_{1}^{*} p_{1}^{*}}{4 C K \theta \omega^{3}}\left(C-a n_{1}^{*}\right)
$$

and because the stationary point $\left(n_{1}^{*}, p_{1}^{*}\right)$ is from the first model, where

$$
n<\frac{C}{a}, \quad \text { then } \quad l_{1}(0)<0
$$

and the limit cycle which appears in this Hopf bifurcation is stable and Hopf bifurcation is supercritical.

For $\left(n_{3}^{*}, p_{3}^{*}\right)$, we have the functions $P\left(\mu_{1}, \mu_{2}\right), R\left(\mu_{1}, \mu_{2}\right)$ :

$$
\begin{aligned}
& P\left(\mu_{1}, \mu_{2}\right)=-\frac{r}{K \theta} \mu_{1}^{3}-\frac{r n_{3}^{*}}{K \theta} \mu_{1}^{2}-\frac{\omega}{n_{3}^{*}} \mu_{1} \mu_{2}, \\
& R\left(\mu_{1}, \mu_{2}\right)=\frac{\alpha a}{2} \mu_{1} \mu_{2} .
\end{aligned}
$$

It is easy to see that the first Lyapunov coefficient is

$$
l_{1}(0)=-\frac{r}{2 K \theta \omega}<0 \text {. }
$$

The limit cycle which appears in this Hopf bifurcation is stable and Hopf bifurcation is supercritical.

Hopf bifurcation corresponding to the stationary point $\left(n_{1}^{*}, p_{1}^{*}\right)$ comes up for

$$
n_{1}^{*}=\frac{K+\theta}{2}
$$

and according to (4) it must hold

$$
K+\theta<\frac{C}{a}
$$

That means the second model has no influence in contrast with Hopf bifurcation corresponding to the stationary point $\left(n_{3}^{*}, p_{3}^{*}\right)$, where because of the influence of the first model it appears the global bifurcation with appearance of the new unstable limit cycle (see Figure 3 ). 


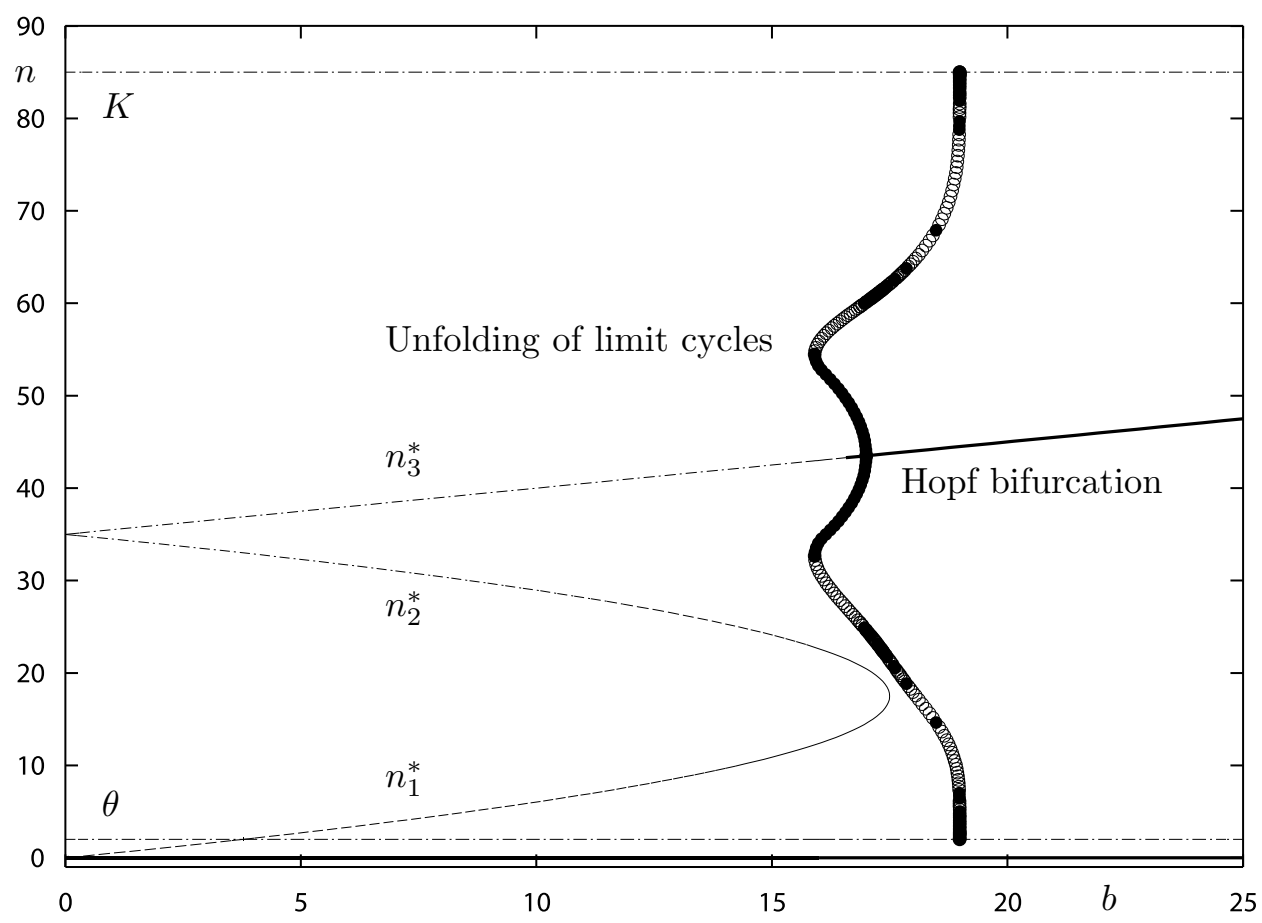

FiguRE 3. Stable cycle branch is presented by filled circles, unstable by unfilled circles. Unstable cycle branch splits on $(\theta, 0)$ and $(K, 0)$.

When

$$
n_{1}^{*}=\frac{C}{2 a}
$$

we get saddle-node bifurcation and in combination with Hopf bifurcation we have Bogdanov-Takens bifurcation. By using analogous normalizing process like in computing of the first Lyapunov coefficient with assuming the condition for $n_{1}^{*}$ we transform the system into the normal form of the Bogdanov-Tankens bifurcation.

We get the eigenvectors of $\boldsymbol{A}_{0}$

$$
\left(\begin{array}{l}
1 \\
0
\end{array}\right), \quad\left(\begin{array}{c}
0 \\
-\frac{1}{a n_{1}^{*}}
\end{array}\right),
$$

matrix $\boldsymbol{T}$

$$
\left(\begin{array}{cc}
1 & 0 \\
0 & -\frac{1}{a n_{1}^{*}}
\end{array}\right),
$$


and functions $P\left(\mu_{1}, \mu_{2}\right), R\left(\mu_{1}, \mu_{2}\right)$ :

$$
\begin{aligned}
& P\left(\mu_{1}, \mu_{2}\right)=-\frac{r \mu_{1}^{3}}{\theta K}+\frac{r\left(K+\theta-3 n_{1}^{*}\right)}{\theta K} \mu_{1}^{2}+\frac{\mu_{1} \mu_{2}}{n_{1}^{*}}+\frac{r}{K \theta}\left(K+\theta-2 n_{1}^{*}\right) n_{1}^{*} \mu_{1}, \\
& R\left(\mu_{1}, \mu_{2}\right)=-\frac{\alpha a^{2} n_{1}^{*}}{2 C}\left[p_{1}^{*}\left(C-2 a n_{1}^{*}\right)-\left(C-2 a n_{1}^{*}\right) \frac{\mu_{2}}{a n_{1}^{*}}-a\left(p_{1}^{*}-\frac{\mu_{2}}{a n_{1}^{*}}\right) \mu_{1}\right] \mu_{1} .
\end{aligned}
$$

The genericity condition for Bogdanov-Tankens bifurcation according to [2] is

where

$$
s=\operatorname{sgn}\left(b_{20}\left(a_{20}+b_{11}\right)\right) \neq 0
$$

$$
\begin{aligned}
& P\left(\mu_{1}, \mu_{2}\right)=a_{20} \mu_{1}^{2}+P_{1}\left(\mu_{1}, \mu_{2}\right), \\
& R\left(\mu_{1}, \mu_{2}\right)=b_{20} \mu_{1}^{2}+b_{11} \mu_{1} \mu_{2}+R_{1}\left(\mu_{1}, \mu_{2}\right) .
\end{aligned}
$$

Since

$$
s=\operatorname{sgn}\left(\frac{\alpha a^{3}}{2 C} n_{1}^{*} p_{1}^{*}\left(-\frac{r n_{1}^{*}}{K \theta}+\frac{\alpha a}{2 C}\left(C-2 a n_{1}^{*}\right)\right)\right)
$$

$s$ is nonzero variable when $\theta \neq K$, which is satisfied since $\theta<K$ in a common situation.

Another Bogdanov-Takens bifurcation is possible when

$$
\lim _{b \rightarrow 0} n_{2}^{*}=\lim _{b \rightarrow 0} n_{3}^{*}=\frac{C}{a}
$$

it is caused by combinations of two models. This implies possibility of splitting of the unstable limit cycle on the saddle point $\left(n_{2}^{*}, p_{2}^{*}\right)$ see Figure 4

Near the Bogdanov-Takens bifurcation there is a unique smooth curve corresponding to a saddle homoclinic bifurcation, where the limit cycle splits on the separatrix loop of the saddle. In our case, see the bifurcation diagram Figure 3, the supercritical Hopf bifurcation curve of the stable limit cycle has a limit point, where the stable cycle turns to be unstable one. It is a typical fold bifurcation of the limit cycles. In a small parameter area there coexist two nearby cycles - stable and unstable one. The unstable one then splits on the separatrix homoclinic loop of one of the saddles (see Figure 3 and 4). 


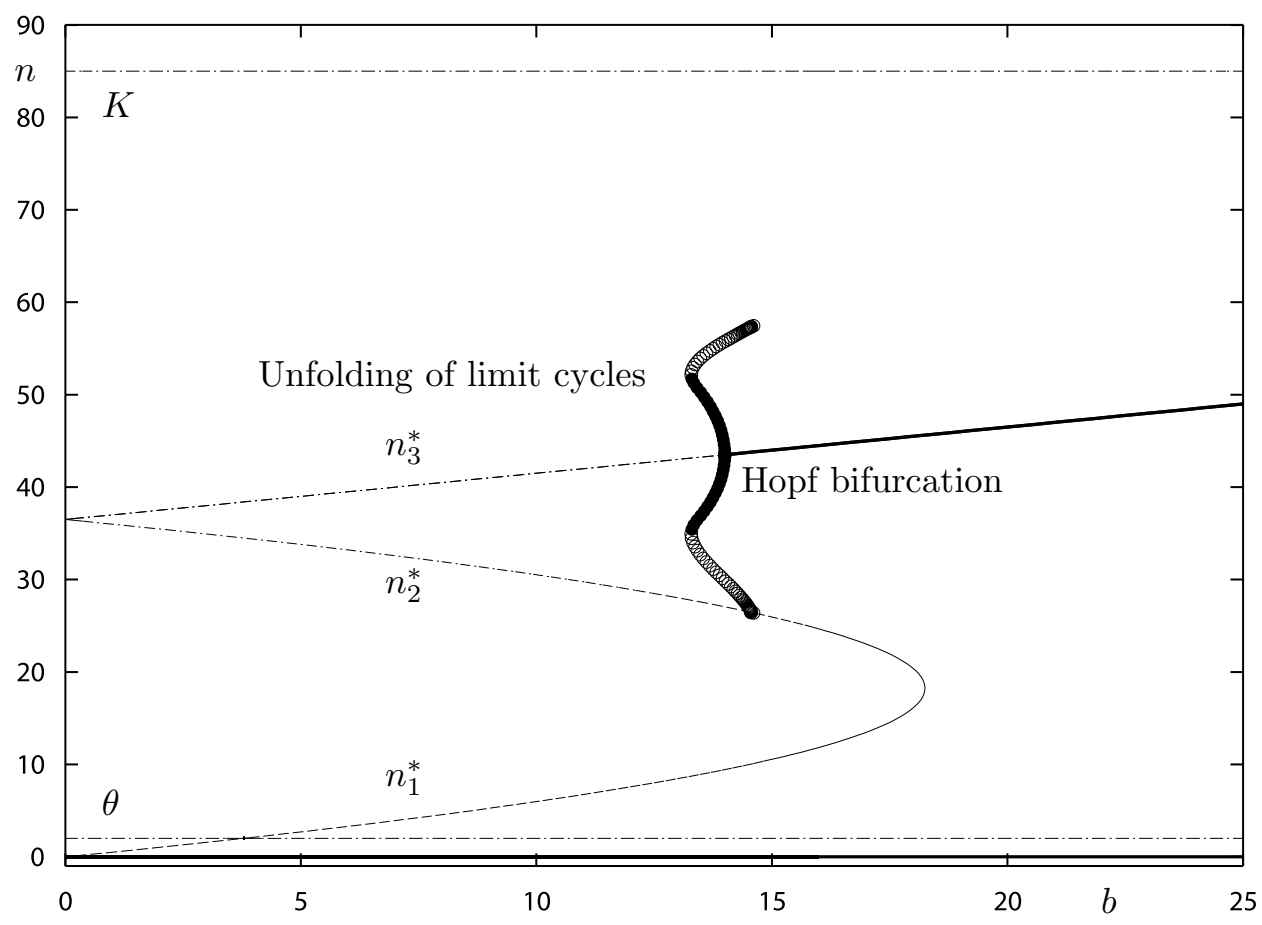

FiguRE 4. Stable cycle branch is presented by filled circles, unstable with unfilled circles. Unstable cycle branch splits on $\left(n_{2}^{*}, p_{2}^{*}\right)$.

Let us look closer to this in some phase portraits (Figure 5 ). As it can be seen in Figure 5 (a) for a small value of parameter $b$ there is a huge unstable area, where both populations become extinct. As the parameter $b$ increases there is unfolding of the stable cycle that arises from the supercritical Hopf bifurcation. This causes appearance of a small stable area "from nothing". When the parameter $b$ crosses the critical value of the local supercritical Hopf bifurcation at the maximum of the parabola nullcline, the stable limit cycle vanishes and the branch of the unstable cycle around the stable focus continues until it splits at the separatrix loop of one of the saddle points. The green area is the basin of attraction of the stable limit cycle or the stable focus, respectively. It is a large area that maintains the populations. On the other hand, decreasing of the value of parameter $b$ (mortality rate of the predator) causes disappearance of the stable green area and there is a global bifurcation - a bluesky catastrophe, extinction of both populations "from nothing". 


\section{A PREDATOR-PREY MODEL WITH ALLEE EFFECT}

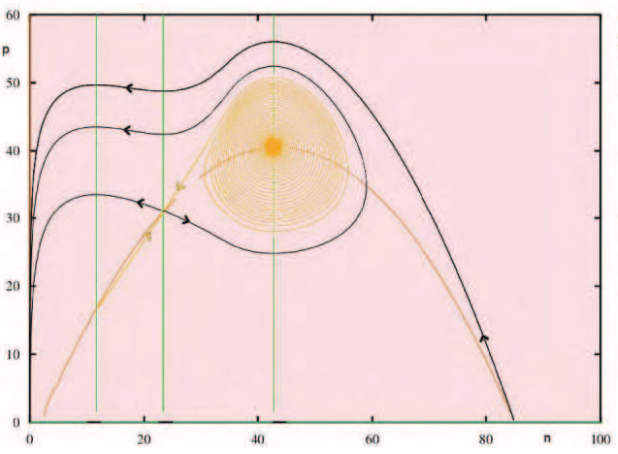

(A) Complete unstable area

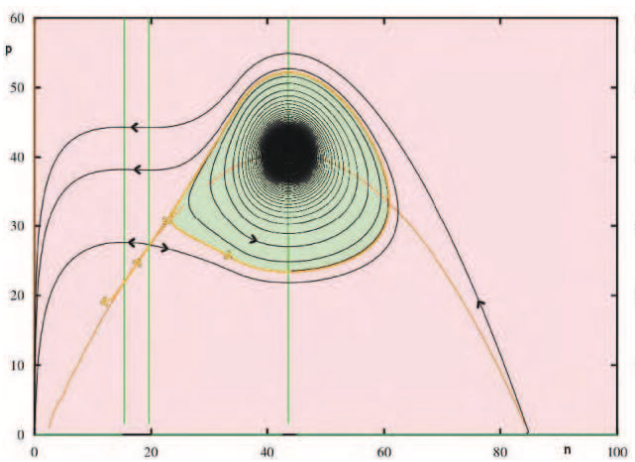

(c) Stable limit cycle vanished, the unstable limit cycle around the stable focus.

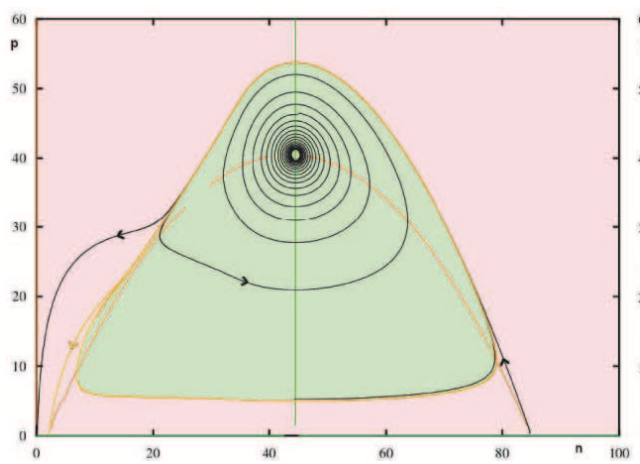

(E) Spreading of the stable area.

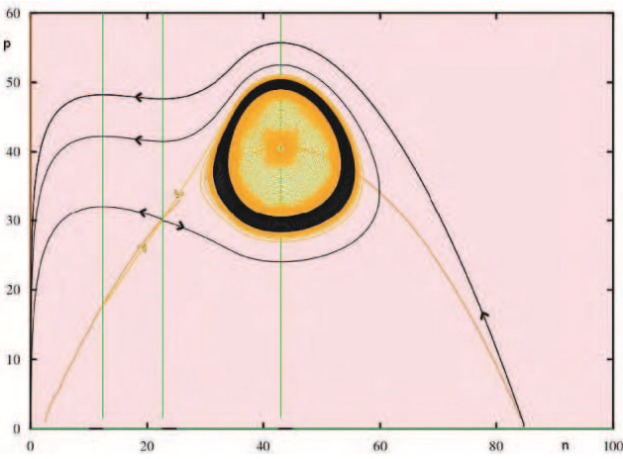

(B) Appearance of the small stable area by unfolding of the limit cycles.

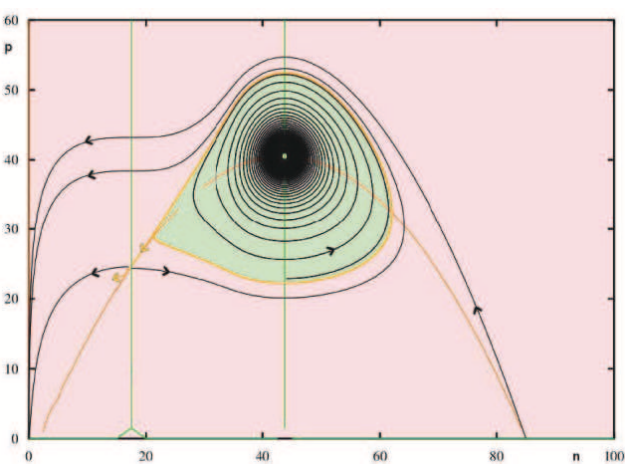

(D) Saddle-node bifurcation of the stationary point $\left(n_{1}^{*}, p_{1}^{*}\right)$ and $\left(n_{2}^{*}, p_{2}^{*}\right)$.

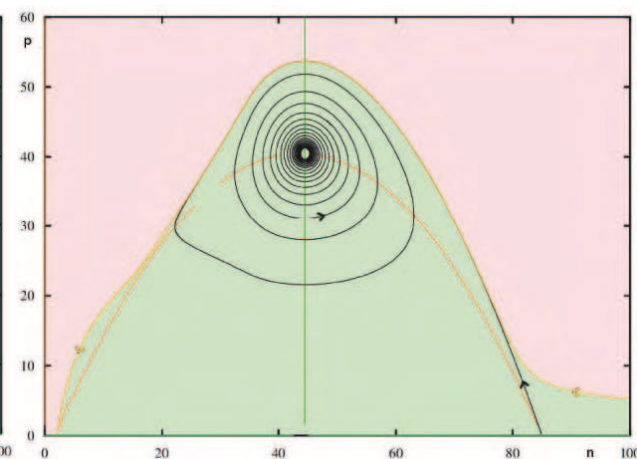

(F) Unstable limit cycle split at the separatrix loop.

FIgURE 5. Phase portraits. 


\section{JITKA KÜHNOVÁ — LENKA PŘIBYLOVÁ}

\section{Conclusion}

In this paper, we have introduced the predator-prey model with Allee effect in prey and in which individual predators can use Hawk and Dove tactics in fighting over caught prey, that caused division into two submodels. We have shown, by bifurcation analysis and respective phase portraits, existence of saddle-node bifurcation, two supercritical Hopf bifurcations and two Bogdanov-Takens bifurcations in this aggregated model. Moreover, we have shown existence of global bifurcation of unfolding of two limit cycles, which is in neither submodel alone.

\section{REFERENCES}

[1] AUGER, P.-PARRA, R. B-MORAND, S.-SÁNCHEZ, E.: A predator-prey model with predators using hawk and dove tactics, Mathematical Biosciences 177\&178 (2002), 185-200.

[2] KUZnetSov, Y. A.: Elements of Applied Bifurcation Theory. Springer-Verlag, Berlin, New York, Inc. 1998.

Received August 8, 2011

Jitka Kühnová

Faculty of Science

University of Hradec Králové

Rokitanského 62

CZ-500-03 Hradec Králové

CZECH REPUBLIC

E-mail: jitka.kuhnova@seznam.cz

Lenka Přibylová

Department of Mathematics and Statistics

Faculty of Science

Masaryk University

Kotlářská 2

CZ-611-37 Brno

CZECH REPUBLIC

E-mail: pribylova@math.muni.cz 